\title{
Roles of NRF2 in Cell Proliferation and Differentiation
}

Shohei Murakami, Hozumi Motohashi

Department of Gene Expression Regulation, Institute of Development, Aging and Cancer, Tohoku University, 4-1 Seiryo-machi, Aoba-ku, Sendai, Miyagi, 980-8575, Japan

CORRESPONDING AUTHOR: Hozumi Motohashi

Hozumi Motohashi (communicating with the Editorial and Production offices) Department of Gene Expression Regulation, Institute of Development, Aging and Cancer, Tohoku University,

4-1 Seiryo-machi, Aoba-ku, Sendai, Miyagi, 980-8575, Japan

TEL: 81-22-717-8550,

FAX: 81-22-717-8554,

E-MAIL: hozumim@idac.tohoku.ac.jp 


\section{Abstract}

The KEAP1-NRF2 system plays pivotal roles in defense mechanisms by regulating cellular redox homeostasis. NRF2 is an inducible transcription factor that activates a battery of genes encoding antioxidant proteins and phase II enzymes in response to oxidative stress and electrophilic xenobiotics. The activity of NRF2 is regulated by KEAP1, which promotes the ubiquitination and subsequent degradation of NRF2 under normal conditions and releases the inhibited NRF2 activity upon exposure to the stresses. Though an impressive contribution of the KEAP1-NRF2 system to the protection from exogenous and endogenous electrophilic insults has been well established, a line of evidence has suggested that the KEAP1-NRF2 system has various novel functions, particularly in cell proliferation and differentiation. Because the proliferation and differentiation of diverse cell types are often influenced and modulated by the cellular redox balance, NRF2 has been considered to control these cellular processes by regulating the cellular levels of reactive oxygen species (ROS). In addition, analyses of the genome-wide distribution of NRF2 have identified new sets of NRF2 target genes whose products are involved in cell proliferation and differentiation but not necessarily in the regulation of oxidative stress. Considering the most characteristic features of NRF2 as an inducible transcription factor, a newly emerged concept proposes that the KEAP1-NRF2 system translates environmental stresses into regulatory network signals in cell fate determination. In this review, we introduce the contribution of NRF2 to lineage-specific differentiation, maintenance and differentiation of stem cells, and proliferation of normal and cancer cells, and we discuss how the response to fluctuating environments modulates cell behavior through the KEAP1-NRF2 system. 
MURAKAMI et al.

Keywords: KEAP1-NRF2 system, cell proliferation, differentiation, stem cells 


\section{Introduction}

The KEAP1-NRF2 system has been known as one of the most critical defense mechanisms against oxidative and electrophilic insults [1]. NRF2 belongs to the Cap'n'collar (CNC) transcription factor family and possesses a unique CNC domain that is linked to the N-terminal side of a highly conserved basic region-leucine zipper (bZIP) motif $[2,3]$. The basic region is for the DNA recognition, and the leucine zipper is for the dimerization with small MAF (sMAF) proteins [4,5]. Because CNC proteins do not bind to DNA as a monomer, sMAF is an obligatory partner molecule that enables the CNC proteins to bind to DNA and exert their function in the transcriptional regulation [6-8]. The recognition sequence of CNC-sMAF heterodimers is called as Maf recognition element (MARE; TGC TGA G/C TCA GCA), but detailed analyses in recent years have revealed that the CNC-sMAF heterodimers preferentially bind to a MARE-related sequence (CNC-sMAF binding element; GC TGA $/ \mathrm{C}$ TCA $/ \mathrm{T}$ ) rather than to the complete canonical consensus MARE [9-12]. The binding sequence of the NRF2-sMAF heterodimer is designated as an antioxidant responsive element (ARE) or electrophile responsive element (EpRE) ( $\mathrm{GC} n n n$ G/C TCA) based on its historical background; it was originally identified as a critical cis-regulatory element in the transcriptional regulation of antioxidant genes [13-15]. A genome-wide collection of the cis-regulatory elements bound by NRF2 is now designated as the "NRF2 cistrome", and the breadth of the "NRF2 cistrome" is much wider than expected and extends into genes that regulate cell proliferation and differentiation (Table 1).

One of the mechanisms by which NRF2 controls cell proliferation and differentiation is the regulation of reactive oxygen species (ROS) (Fig. 1A). ROS have been recently recognized as one of important signal mediators [16-18]. ROS includes 
MURAKAMI et al.

the initial species formed by reduction of oxygen, like superoxide anion, hydroxyl radical, hydrogen peroxide $\left(\mathrm{H}_{2} \mathrm{O}_{2}\right)$, but also encompass other radical and non-radical secondary reactive products generated by reaction with the initial species $[19,20]$. In this review, we utilize the term "ROS" to indicate all these reactive species that may include experimentally-unidentified reactive products, and instead of identifying which chemical compounds participate in the signal transduction, we describe how ROS are measured in each study. ROS-mediated signaling pathways are heavily dependent on the availability of thioredoxin and glutathione [19,20]. NRF2 increases cellular amounts of reduced thioredoxin and glutathione through upregulating the enzymes involved in their reduction and synthesis, resulting in elimination of ROS in general [21]. This is the way in which NRF2 utilizes ROS as a mediator to transmit the NRF2-activating signals to cellular programs that modify proliferation and/or differentiation.

Other mechanisms are not necessarily related to the regulation of oxidative stress (Fig. 1B). Due to the recent technical advances, the genome-wide distribution of NRF2 has been clarified in several contexts and has led to the identification of novel NRF2 target genes. In this case, NRF2 directly activates the genes that are involved in the regulation of cell fate determination.

Considering that NRF2 is an inducible transcription factor whose activity is regulated by KEAP1 in response to electrophiles, a novel concept emerges in which the KEAP1-NRF2 system translates the oxidative and electrophilic stresses originating from exogenous and endogenous sources into regulatory network signals that influence cell fate determination. In this review, we introduce the NRF2 contribution to the lineage specification, the maintenance and differentiation of stem cells, and the proliferation of normal and cancer cells. We also discuss how the response to fluctuating environments 
modulates the processes of cellular development through the KEAP1-NRF2 system.

\section{Regulation of cell differentiation}

\section{1) Osteoclastogenesis and osteoblastogenesis}

Although ROS were once considered to be toxic pathological compounds that cause cellular damage and dysfunction, recent studies have clarified the physiological roles of ROS as signaling mediators that regulate various cellular functions, including proliferation and differentiation [16-18]. Osteoclasts and osteoblasts are two major players involved in the maintenance of bone homeostasis, where ROS mediate physiological signaling.

Bone homeostasis is maintained based on the balance between formation and resorption mediated by osteoblasts and osteoclasts, respectively, and its imbalance results in pathological conditions such as rheumatoid arthritis and osteoporosis [22]. Osteoclasts are derived from hematopoietic stem cells (HSCs), and osteoblasts differentiate from mesenchymal stem cells (MSCs). The development and differentiation of these two distinct cell types are controlled by a number of cytokines and growth factors. Well-coordinated actions of these factors are essential for the appropriate generation of osteoblasts and osteoclasts and thereby the maintenance of the sophisticated balance between bone formation and bone resorption.

In addition to these classical humoral factors, osteoclast differentiation is modulated depending on the intracellular ROS, particularly $\mathrm{H}_{2} \mathrm{O}_{2}[23,24]$. A murine macrophage cell line (RAW264.7 cell) and bone-derived macrophages efficiently differentiate into osteoclasts in response to stimulation with RANK-L, and the intracellular amounts of ROS become elevated as they differentiate. Interestingly, 
MURAKAMI et al.

attenuated NRF2 activity increases the accumulation of ROS measured by dye oxidation protocol such as dichlorodihydrofluorescein diacetate $\left(\mathrm{H}_{2}\right.$ DCFDA) along with decrease in reduced glutathione, and enhances osteoclast differentiation [25,26] (Fig. 1A (i)). Treatment of osteoclast precursor cells with $\mathrm{N}$-acetylcysteine (NAC), an antioxidant agent, inhibits osteoclast differentiation, which indicates that increased ROS facilitate osteoclastogenesis in the absence of NRF2.

While NRF2 activation inhibits osteoclast differentiation by reducing the intracellular ROS, osteoblast differentiation is also inhibited by NRF2, but this is not dependent on redox regulation in this case. It has been shown that NRF2 directly associates with RUNX2, which is a key regulator of the osteoblast differentiation [27,28], and suppresses its transcriptional activity [29]. Interestingly, the interaction between NRF2 and RUNX2 is likely to impair the differentiation of chondrocytes as well. Similar to osteoblasts, chondrocytes are derived from MSCs, and RUNX2 plays a pivotal role in chondrogenesis [30]. NRF2 overexpression in the ATDC5 pre-chondrogenic cell line inhibits chondrocyte maturation [31].

As mentioned above, NRF2 inhibits the differentiation of both osteoclasts and osteoblasts, which raises a question of how NRF2 contributes to bone homeostasis in vivo. There is a study answering to the question by demonstrating that the inhibitory effect of NRF2 is dominant in the osteoblast differentiation in vivo [32]. Compared with wild-type mice, $N r f 2$-deficient mice exhibit an increase in bone mass due to a larger number of osteoblasts. Whereas NRF2 disruption enhances osteoclastogenesis in vitro under RANK-L stimulation, no functional enhancement of osteoclasts is apparent in Nrf2-deficient mice. This discrepancy is due to osteoprotegerin (OPG), which is a decoy receptor of RANK-L that is secreted from osteoblasts in vivo. Osteoclastogenesis is 
accomplished through the fine balance between RANK-L and OPG, and a predominance of OPG inhibits osteoclast differentiation [33,34]. The study suggests that NRF2 negatively regulates the expression of OPG in osteoblasts through direct binding to AREs in the promoter region of the OPG gene, although NRF2 usually serves as a transcription activator [32]. Therefore, it is likely that NRF2 deficiency enhances OPG expression and thereby suppresses osteoclastogenesis in vivo.

In contrast, under stressed conditions, the impact of NRF2 deficiency is more obvious in osteoclasts than osteoblasts. Ionizing radiation (IR) generates oxidative stress, which is implicated as a cause of IR-induced osteoporotic phenotypes $[35,36]$. The bone loss initiated by IR is augmented in Nrf2-deficient mice, which is accompanied by a higher number of osteoclasts than wild-type mice [37]. Interestingly, Nrf2-deficient osteoblasts increase the RANK-L expression after IR, presumably resulting in osteoclast expansion.

Overall, the impacts of the NRF2 deficiency on bone homeostasis, especially the behavior of osteoclasts and osteoblasts, seem to be context dependent. Environmental stress, i.e., irradiation, changes the contribution of NRF2 to the maintenance of bone homeostasis.

\section{2) Adipogenesis and obesity}

Adipocyte differentiation is another well-studied system in the context of the association between NRF2-dependent transcription networks and cell maturation, and it is closely associated with the pathogenesis of diabetes. Two factors responsible for adipogenesis are directly regulated by NRF2 [38,39] (Fig. 1B (i)). One is CCAAT/enhancer-binding protein $\beta$ (CEBP $\beta)$, which is transiently expressed at the onset of adipocyte 
MURAKAMI et al.

differentiation and initiates the differentiation programs. The other is peroxisome proliferator-activated receptor $\gamma(\operatorname{PPAR} \gamma)$, which orchestrates the differentiation process downstream of CEBP $\beta$ [40]. NRF2 regulates these two genes through binding to the AREs located in each promoter region, and its defect suppresses adipogenesis in vitro [38,39]. Supporting this in vitro observation, either global or adipocyte-specific disruption of $N r f 2$ gene reduces white adipose tissue mass in $o b / o b$ mice, which is an obese mouse model characterized by leptin deficiency [41]. The $o b / o b$ mice with adipocyte-specific disruption of Nrf2 exhibit aggravation of insulin resistance, hyperglycemia and hypertriglyceridemia, which may be associated with the limited deposition of lipids in the Nrf2-deficient adipose tissues. These studies support the notion that NRF2 promotes adipocyte development.

Whereas NRF2 deficiency impairs adipocyte development, NRF2 activation also decreases adipose tissue mass and lipid accumulation in the $o b / o b$ mice and limits the induction of adipocyte differentiation from embryonic fibroblasts (MEF) in vitro [42]. Similarly, previous studies have shown that NRF2 inhibits adipocyte differentiation by directly activating the transcription of the aryl hydrocarbon receptor $(A h R)$ gene or the retinoid X receptor $\alpha($ Rxra $)$ gene, which are involved in lipid metabolism [43,44] (Fig. 1B (ii)). AHR is decreasingly expressed during adipogenesis, and the forced expression of AHR inhibits the differentiation of MEFs into adipocytes [43]. Thus, AHR is one of the candidates downstream effectors of NRF2 in adipogenesis inhibition. RXR $\alpha$ is another candidate and exerts repressive effects on adipogenesis by changing the $\mathrm{RXR} \alpha / \mathrm{RXR} \beta$ ratio that modulates PPAR $\gamma$ activity [45]. Therefore, the NRF2-mediated upregulation of the Rxra gene appears to perturb the fine tuning of the $\mathrm{RXR} \alpha / \mathrm{RXR} \beta$ ratio during adipogenesis. 
Similar to the NRF2 functions in bone homeostasis, an in vitro differentiation system examining cell-autonomous roles of NRF2 has shown the context-dependent contribution of NRF2 to adipogenesis. In addition to the cell-autonomous effects of the NRF2 activity, adipocyte development and lipid deposition in adipocytes are heavily influenced by the systemic energy metabolism, involving skeletal muscles, liver and pancreas. In particular, the balance between energy intake and expenditure is one of the most critical determinants of adipose tissue mass. A recent investigation has revealed that systemic activation of NRF2 due to Keapl gene knockdown improves insulin resistance and prevents obesity induced by a high-fat diet [46]. Another study demonstrated that selective activation of NRF2 in pancreatic $\beta$ cells robustly preserves $\beta$ cells in the iNOS-Tg diabetes mouse model and mitigates the progression of diabetes [47]. Thus, these cell-autonomous and non-cell-autonomous factors would be integrated into the observable NRF2 functions in adipogenesis in vivo.

\section{3) Spermatogenesis and other tissue development}

It is known that spermatozoa are highly susceptible to oxidative stress and need to be protected by sufficient amounts of antioxidants in the reproductive tract $[48,49]$. Consistent with this notion, spermatogenesis is closely associated with NRF2 activity. Nrf2-deficient male mice exhibit decreased fertility due to accumulating damage to germ cells with increasing age [50]. Testicular and epididymal glutathione and antioxidant enzyme activities are significantly lower in Nrf2-deficient mice than in wild-type mice. Conversely, lipid peroxidation products are elevated in Nrf2-deficient testes and epididymides. These results indicate that NRF2 prevents oxidative stress in spermatozoa and supports spermatogenesis. 
MURAKAMI et al.

Intriguingly, single nucleotide polymorphisms (SNPs) in the human NRF2 gene promoter region have been found to associate with idiopathic oligoasthenozoospermia [51]. One of the SNPs, rs6721961, corresponds to a critical determinant of the transcription levels of the NRF2 gene, and the decreased NRF2 expression due to the SNP is significantly associated with a higher frequency of lung cancers in smokers [52]. Therefore, it is plausible that the low NRF2 activity that results from the SNPs causes spermatozoa to be highly susceptible to oxidative damage and consequently results in defective spermatogenesis.

In addition to cell differentiation, the NRF2-mediated modification of tissue development has been observed in lung development and epidermal barrier formation [53,54]. NRF2 prevents neonatal pulmonary cells from hyperoxic cell death [53]. NRF2 also compensates for defective epidermal barrier formation in the absence of loricrin, a glycine-/serine-rich structural protein in a cornified envelope [54]. In both cases, NRF2 is vital for tissue development in the presence of disruptive factors originating from exogenous or endogenous sources, whereas NRF2 is dispensable under non-stressed conditions. In summary, NRF2 guarantees a normal process of tissue development by maintaining the redox homeostasis in response to environmental fluctuations and also transmits the environmental signals to the molecular machinery that regulates the differentiation process by directly controlling tissue-specific regulators of differentiation.

\section{Contribution to stem cell maintenance and cell fate determination}

\section{1) Interaction between NRF2 activity and ROS in stem cells}

Stem cells are characterized by self-renewal ability and multipotency, and most of them 
are often maintained in quiescence under normal conditions. The dormancy is beneficial for stem cells to avoid unnecessary DNA replication and eventual exhaustion by reducing the chance of DNA damage and mutations [55-58]. Recently, ROS have been suggested as one of the important regulators of stem cell self-renewal [59]. However, the roles and functions of ROS in stem cells are diverse and presumably context dependent [60-62]. In some cases, stem cells are stimulated to proliferate by increased ROS including $\mathrm{H}_{2} \mathrm{O}_{2}$ that are a major origin of ROS signaling pathways [60,61], but in other cases, ROS accumulation deteriorates stem cell functions and maintenance, which can be rescued by NAC treatment [62-64]. Thus, it is not surprising that observable contributions of the KEAP1-NRF2 system to stem cell activity also vary from study to study depending on experimental systems.

It has been reported that $\mathrm{CncC}$, which is a Drosophila homolog of NRF2, is constitutively active in intestinal stem cells (ISCs) of Drosophila and maintains ISCs quiescent with low ROS concentration [65] (Fig. 1A (ii)). Interestingly, for the ISC proliferation that is induced by an increase in ROS accumulation, the KEAP1-mediated inhibition of $\mathrm{CncC}$ is required. In this experimental setting, it is unclear what reactive components are the substantial mediators, because ROS were measured by dye oxidation protocol using dihydroethidium (DHE), which has been suggested to have several limitations [66,67]. However, overexpression of gclc, the rate-limiting enzyme for glutathione synthesis, and jafracl, a thioredoxin peroxidase, leads to decrease in the KEAP1-mediated ISC proliferation along with reduced levels of oxidative stress. Therefore, it is interpreted that ROS are a downstream effector of the KEAP1-NRF2 system, and that the ROS elevation caused by the $\mathrm{CncC}$ repression drives the ISC proliferation. 
MURAKAMI et al.

In other cases, ROS are not necessarily a major downstream effector of NRF2 in stem cell proliferation. In bronchial epithelia, airway basal stem cells (ABSCs) play important roles in the maintenance of airway homeostasis $[68,69]$. When the tracheobronchial epithelium is injured by environmental hazards or stress, ABSCs are driven into proliferation and differentiation to replenish epithelial cells. There is a positive correlation between the ROS concentration and the ABSC proliferation [70]. Exposure of ABSCs to exogenous $\mathrm{H}_{2} \mathrm{O}_{2}$ increases the intracellular concentration of ROS, which is defined by $\mathrm{H}_{2}$ DCFDA, DHE, and Mito Sox, and accelerates the ABSC proliferation, whereas the NAC treatment takes the opposite effect. The NRF2 activity is enhanced as the ROS increase, implying that NRF2 may be activated in response to the increase in ROS [70]. Importantly, downstream of the ROS-mediated NRF2 activation, NOTCH1 is responsible for the proliferation of ABSCs (Fig. 1B (iii)). The introduction of the intracellular domain of NOTCH1 almost completely rescues the impaired ABSC proliferation in the absence of NRF2. Therefore, for ABSC proliferation, NRF2 conveys the ROS signals to the NOTCH1 pathway.

\section{2) NRF2-dependent gene regulation in stem cells and progenitor cells}

There are some situations in which NRF2 regulates the maintenance and differentiation of stem/progenitor cells through mechanisms that are unrelated to intracellular ROS control. One example is found in the control of self-renewal and pluripotency of human embryonic stem cells (hESCs) by NRF2 [71]. NRF2 is highly expressed in undifferentiated hESCs compared with differentiated cells, implying the functional significance of NRF2 in the pluripotent status of hESCs. Indeed, a reduction of NRF2 activity enhances the differentiation of hESCs, and the maintenance of pluripotency is 
inhibited by $N r f 2$ knockdown. A decrease in proteasome activity induced by the NRF2 knockdown has been suggested to be an underlying mechanism of the impairment of pluripotency because the sufficient levels of proteasome activity is essential for the maintenance of pluripotency. Among the genes required for appropriate proteasome activity, POMP, a chaperone for the assembly of proteasomal subunits, is one of the candidates that fall under the control of NRF2. Taken together, NRF2 is at least partly responsible for the maintenance of hESCs through modulation of proteasome activity.

Another example is found in satellite cells (SCs) that form a resident population of stem/progenitor cells in skeletal muscles. SCs are maintained in a quiescent state outside the cell cycle under normal conditions. Upon the skeletal muscle injury, they begin to proliferate and differentiate, which is an essential process of muscle repair [72,73]. The proliferation and differentiation of SCs are induced by myogenic regulatory factors, MyoD and myogenin, respectively [72,73]. During muscle regeneration, NRF2 supports the efficient proliferation of SCs by upregulating MyoD and suppressing myogenin expression [74]. Consequently, Nrf2 deficiency delays muscle regeneration after the injury. Because MyoD has a well-conserved functional ARE in its promoter region, it appears to be a direct target of NRF2 in the activation of dormant SCs during the injured muscle repair (Fig. 1B (iv)).

Neuronal progenitor cells (NPCs) still provide another example in which NRF2-dependent gene regulation accounts for its regenerative activity. Increased NRF2 activity enhances both proliferation and differentiation of NPCs, and functional impairment of NRF2 retards compensatory neurogenesis in a global brain ischemia model [75]. Consistent with this observation, NRF2 activation potentiates retinoic acid-induced neuronal differentiation of a neuroblastoma cell line, SH-SY5Y cells, 
MURAKAMI et al.

whereas the NRF2 activation itself is insufficient to stimulate the differentiation [76].

We can deduce from these examples that NRF2 plays distinct roles in pluripotent stem cells and in tissue stem cells. In hESCs, NRF2 inhibits differentiation but contributes to the maintenance of pluripotency [71]. In SCs and NPCs, which are categorized into tissue stem cells, NRF2 enhances proliferation and/or differentiation for tissue regeneration $[74,75]$. In the following section, we focus on NRF2 functions in hematopoietic stem cells (HSCs), which are one of the best-characterized tissue stem cells.

\section{3) Roles of NRF2 in hematopoietic stem cells (HSCs)}

Due to the well-established transplantation technique, the characterization of HSCs is much more advanced than that of other tissue stem cells. Most HSCs are maintained in a quiescent state and hardly contribute to daily hematopoiesis $[77,78]$. However, under stressed conditions, e.g., during inflammation or after bone marrow transplantation, HSCs proliferate, increase in number and produce progenitor cells and, subsequently, mature cells [77-80]. Several studies have shown that HSCs are vulnerable to oxidative stress $[63,64,81]$; thus, to escape from oxidative stress, HSCs are localized in special environments, called "niches", where the oxygen levels are relatively low [62,82]. For example, when the genes essential for HSC function and maintenance are deleted in HSCs, such as FOXOs and TSC1, accumulation of ROS is observed $[63,81,83]$. While it is obscure what reactive species are responsible for the HSC exhaustion, ROS are considered as major causes for HSC exhaustion, based on the fact that the HSC impairment is partially restored by NAC treatment $[63,81,83]$.

The results of these reports lead to the expectation that the KEAP1-NRF2 system 
engages in the control of HSCs. Indeed, $N r f 2$ deficiency attenuates the reconstitution capacity of HSCs after the transplantation, indicating that NRF2 is required for the HSC maintenance $[84,85]$. Of note, this effect of NRF2 on the HSC maintenance is independent of cellular ROS concentration [84]. One study suggests that NRF2 directly binds to ARE in the promoter region of Cxcr4 [85], which is indispensable for the maintenance of the quiescent HSC pool [86] (Fig. 1B (iv)). The reduced CXCR4 signaling disturbs the maintenance of HSCs in the absence of NRF2 [85].

In addition to the Nrf2-deficient model, Keap1-deficient HSCs, in which NRF2 is constitutively activated, were examined to clarify the contribution of NRF2 activation to the stem cell function [87]. Keapl deficiency in HSCs mitigates irradiation-induced myelosuppression and promotes the early reconstitution of bone marrow cells after transplantation. The enhancement of HSC activity is attributed to the upregulation of the NOTCH1 pathway, as observed in ABSCs (Fig. 1B (iii)). These results clarify a benefit of NRF2 activation in HSCs for short-term reconstitution. However, further analyses are required to understand the contribution of NRF2 to the long-term reconstitution ability of HSCs.

The KEAP1-NRF2 system also contributes to the differentiation as well as maintenance of HSCs. Recently, we found that NRF2 activation skews the differentiation potential of HSCs and facilitates granulocyte/monocyte production at the expense of erythroid and lymphoid cells [88]. In Keapl-deficient hematopoietic cells, the expression levels of genes that are important for lineage specification are already altered at the stage of HSCs from erythroid and lymphoid to myeloid dominance, and the skewing is restored by simultaneous disruption of $N r f 2$ on the Keapl-null background. Therefore, NRF2 activation in HSCs drives the myeloid differentiation and represses 
MURAKAMI et al.

erythroid and lymphoid differentiation.

The effect of NRF2 on hematopoietic differentiation has been demonstrated not only in normal HSCs but also in acute myeloid leukemic (AML) cells [89]. It has been shown that NRF2 activation enhances the 1,25D (1 $\alpha, 25$-dihydroxyvitamin $\left.\mathrm{D}_{3}\right)$-induced differentiation of AML cells by increasing the activity of activator protein-1 (AP-1) and the subsequent elevation of vitamin D receptor and RXR $\alpha$ expression. The activation of NRF2, which drives the maturation of leukemic cells beyond the differentiation block, might be a promising strategy for differentiation therapy of leukemia.

\section{4) Roles of NRF2 in other hematopoietic cell lineages}

Given that NRF2 is expressed throughout the differentiation stages of hematopoietic cells, NRF2 contributes broadly to the differentiation and maturation of more differentiated cells, such as erythrocytes and megakaryocytes [90-92]. NRF2 was initially identified as one of the regulators of erythroid-specific genes, such as the $\beta$-globin gene [93], but early studies concluded that NRF2 was not essential for erythroid production because Nrf2-deficient mice did not display any apparent defects in erythropoiesis [94-96]. However, this conclusion has been recently challenged by detailed analyses of

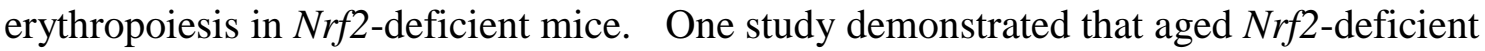
mice exhibit a decreased red blood cell count as a result of immune-mediated hemolysis [90]. Nrf2-deficient erythrocytes are morphologically abnormal and are more sensitive to $\mathrm{H}_{2} \mathrm{O}_{2}$-induced hemolysis than are wild-type erythrocytes. Due to an increased level of oxidative damage, an increased number of erythrocytes are bound by $\operatorname{IgG}$ in Nrf2-deficient mice, which results in the immune-mediated hemolysis. In support of this study, Nrf2 has been found to be essential for the protection of erythrocytes from 
hemolytic anemia by regulating redox homeostasis in erythrocytes [91]. When the synthesis of antioxidant selenoproteins is defective, NRF2 makes a larger contribution to erythrocyte protection than under normal conditions and compensates for the impaired ROS scavenging activity. Thus, NRF2 plays a critical role in the maintenance of redox homeostasis in erythrocytes to prevent hemolytic anemia, while NRF2 activation limits the production of erythrocytes [88].

In megakaryopoiesis, NRF2 is involved in the regulation of megakaryoblast proliferation and megakaryocyte maturation by modulating the activity of NF-E2 p45 [92], which is a key regulator of megakaryopoiesis and platelet production [12,97,98]. NRF2 and NF-E2 p45 both belong to the CNC transcription factor family and share the CNC-sMAF binding elements [3,4,99]. In immature megakaryoblasts, NRF2 is dominant and promotes their proliferation, whereas NF-E2 p45 dominates over NRF2 and outcompetes NRF2 for the binding sites in mature megakaryocytes [92]. Because the transcriptional activation ability of NF-E2 p45 is weaker than that of NRF2, occupancy of the AREs by NF-E2 p45 limits the expression of antioxidant proteins and consequently increases the intracellular concentration of ROS, which is critical for the terminal maturation of megakaryocytes and platelet production. According to the fact that inhibition of NADPH oxidases impairs the maturation of megakaryocytes, particularly polyploidisation, superoxide anions produced by NADPH oxidases are one of essential components for megakaryocyte differentiation [100]. Megakaryocytes provide a prototype demonstrating the significance of functional balance among CNC family proteins in transcriptional regulation via the CNC-sMAF binding elements.

Other CNC family members, including NRF1, NRF3, BACH1 and BACH2, are also considered to share the DNA binding site in principle [101-104]. Competitive 
MURAKAMI et al.

relations have been reported between NRF2 and $\mathrm{BACH} 1$ in the regulation of $\mathrm{Ho}-1$ gene in various cell lineages $[104,105]$ and between NRF2 and NRF1 in the regulation of xCT gene in liver [106]. In addition, a recent report has suggested an intriguing possibility that the balance between $\mathrm{NRF} 2$ and $\mathrm{BACH} 2$ regulates the myeloid and lymphoid differentiation [107]. $\mathrm{BACH} 2$ is a key regulator of $\mathrm{B}$ cell development and maturation [108], and its deficiency enhances myeloid-specific gene expression and skews the differentiation of progenitor cells toward the myeloid lineage [107]. Considering that NRF2 activation promotes myeloid differentiation at the expense of lymphoid differentiation [88], this lineage skewing in Bach2-deficient mice might be mediated by NRF2.

\section{5) Cancer stem cells and Nrf2 activation}

Cancer stem cells (CSCs) are considered to be a rare subpopulation that exhibits high resistance to radiotherapy and chemotherapy $[109,110]$. Their therapeutic resistance is mostly attributable to cell cycle quiescence and to the elevated expression of antioxidant proteins. Because NRF2 is a master regulator of genes encoding antioxidant proteins, detoxification enzymes and drug transporters, NRF2 activity is likely to account for the features of CSCs.

Recent cancer genome analyses have identified somatic mutations in the KEAP1-NRF2 pathway in various human cancers, especially in lung, bladder, and head and neck [111-113]. In many cases, the mutations in KEAPl and NRF2 genes result in constitutive activation of NRF2 and elevated expression of cytoprotective genes [114-116]. Higher and lower NRF2 activity is associated with a worse and better prognosis of patients, respectively [116-120], which suggests critical roles for NRF2 
target gene products in the acquisition of tumorigenicity and therapeutic resistance. Among numerous studies on NRF2 activation in cancers, some reports have elaborated on the NRF2 functions in CSCs.

In glioma stem cells (GSCs), which are one of the best-studied CSCs [121,122], NRF2 has been found to be necessary for their self-renewal capacity [123]. Consistently, NRF2 expression is higher in GSCs than non-stem-like fractions [124]. Another report demonstrated that the induction of an NRF2-dependent transcriptional network supports the survival and self-renewal of GSCs when they are challenged with anticancer agents [125].

Significant roles of NRF2 have been also described from the viewpoint of de-differentiation of cancer cells. The de-differentiation of cancers, which is often mediated by epithelial-to-mesenchymal transition, confers drug resistance and CSC-like properties. NRF2 has been shown to be activated in the de-differentiated cells via phosphorylation by ER membrane kinase PERK, eliciting the expression of multidrug resistant genes [126].

Overall, NRF2 contributes to the survival, self-renewal and acquisition of therapeutic resistance of CSCs, resulting in the malignant behavior of cancers and poor clinical outcomes.

\section{Contribution to cell proliferation}

\section{1) Interaction with the PI3K-AKT pathway}

Though the beneficial effects of NRF2 activation in tissue regeneration have been explained here so far through the NRF2 functions in tissue stem cells, NRF2 also promotes the proliferation of differentiated cells. The liver has high regenerative activity, 
MURAKAMI et al.

and liver regeneration provides an ideal experimental system for examining the NRF2 contribution to reactive cell proliferation. Nrf2-deficient mice exhibit a significant delay in liver regeneration after partial hepatectomy, with a decreased number of proliferating hepatocytes and an increased number of apoptotic hepatocytes [127]. The amount of ROS, determined by $\mathrm{H}_{2}$ DCFDA and DHE, is also higher in the $N r f 2$-deficient hepatocytes than in wild-type control. Given that tumor necrosis factor $\alpha$-induced ROS production provokes apoptosis in hepatocytes [128], the NRF2 deficiency seems to cause the ROS accumulation, which results in the increased apoptotic hepatocytes in the regenerating liver. Insufficient NOTCH1 signaling is proposed as another cause of the delayed liver regeneration in Nrf2-deficient mice because Notchl is a direct target of NRF2 [129] (Fig. 1B (iii)). Supporting this concept, the forced expression of the intracellular domain of NOTCH1 rescues the delayed liver regeneration in $\mathrm{Nrf2}$-deficient mice. Conversely, NRF2 activation enhances reactive proliferation in hepatocytes after portal vein branch ligation [130].

During liver regeneration due to partial hepatectomy, the activation of the PI3 kinase (PI3K)-AKT signaling pathway was attenuated in Nrf2-deficient mice, suggesting a novel role of NRF2 in the regulation of growth factor signaling [127]. We employed an alternative approach and showed a reciprocal activation loop between NRF2 and the PI3K-AKT signaling pathway [131,132]. Liver-specific disruption of Keapl stabilizes NRF2, and liver-specific Pten activates the PI3K-AKT pathway and increases the phosphorylation level of GSK3. Notably, liver-specific Keapl and Pten double disruption results in the massive accumulation of NRF2 with robust activation of NRF2 target genes and a remarkable increase in the phosphorylation of AKT and GSK3 [132]. The increased phosphorylation of AKT and GSK3 is both canceled by the additional 
disruption of $N r f 2$, indicating that NRF2 activation is responsible for the augmentation of PI3K-AKT signaling in the double mutant liver. Elucidation of the mechanisms underlying the potentiation of PI3K-AKT signaling by NRF2 requires further investigation.

In contrast, the enhancement of NRF2 activity by active PI3K-AKT signaling is explained by the inactivation of a KEAP1-independent degradation system of NRF2. Recent studies have clarified a novel mechanism of NRF2 degradation, which is mediated by CUL1- $\beta$ TRCP E3 ubiquitin ligase in cooperation with GSK3 [133,134]. Once phosphorylated by GSK3, NRF2 is trapped and ubiquitinated by CUL1- $\beta$ TRCP complex and is finally degraded. In the sustained activation of PI3K-AKT pathway, GSK3 is phosphorylated and inactivated, which leads to the shutting off of the CUL1- $\beta$ TRCP-mediated degradation of NRF2. Importantly, the inactivation of the KEAP1-independent degradation takes effect in the absence of KEAP1 [132], suggesting that the NRF2 that escapes from the KEAP1-dependent degradation becomes a substrate of the KEAP1-independent degradation system.

Massive accumulation of NRF2 in Keapl::Pten double deficient liver causes remarkable hepatomegaly with an aberrant increase in tubular structures composed of cholangiocytes [132]. An interesting connotation that has emerged from this result is that NRF2 not only promotes proliferation of liver cells but also influences the lineage commitment between hepatocytes and cholangiocytes. Taken together, it is plausible that NRF2 establishes a crosstalk with the PI3K-AKT pathway to regulate cell growth and fate decisions in various tissues.

\section{2) Regulation of cell cycle-related factors}


MURAKAMI et al.

ChIP-sequencing analyses for the genome-wide distribution of NRF2 in combination with gene expression analyses have identified novel sets of NRF2 target genes [11,44,135]. For instance, proliferation-associated genes, including Bmprla, Ifg 1, and Jag1, have been identified as direct targets of NRF2 [135]. The list of NRF2 target genes includes Cdkn1a (p21) and $C d k n 2 b$ (p15), which are inhibitory cell-cycle regulators. These genes may be related to stress-induced cell cycle arrest in the NRF2-mediated response to oxidative stress.

It has been described that NRF2 is required for the transition from $\mathrm{G} 2$ phase to $\mathrm{M}$ phase in the cell cycle, based on the observation that functional impairment of NRF2 compromises or delays M-phase entry [136,137]. The G2/M transition is driven by the activation of Cdk1/Cyclin B1 pathway, and the Cdk1/Cyclin B1 complex activity is diminished by the phosphorylation of Cdk1 by Wee1 kinase $[138,139]$. The accumulation of Wee1 and hyperphosphorylation of Cdk1 is observed in Nrf2-deficient hepatocytes during liver regeneration, which presumably causes G2/M arrest [136]. Another study proposes that glutathione is a substantial modifier of cell cycle status downstream of NRF2 because the G2/M arrest caused by the NRF2 deficiency is rescued by glutathione supplementation, but interestingly not by NAC [137]. These results suggest that NRF2 activity is closely associated with cell cycle regulation.

\section{3) Regulation of cellular metabolism}

It has been well established that higher activity of NRF2 in cancer tissues is strongly correlated with poor prognosis for patients [116-120]. This is not difficult to understand, considering that NRF2 confers resistance against chemotherapy and radiotherapy by coordinately activating cytoprotective genes. In addition, one study has provided a new 
concept of the roles of NRF2 in cancer malignancy by demonstrating that KRAS-mediated carcinogenesis and cancer progression is inhibited in Nrf2-null mice [140]. Their finding implies that NRF2 is required in the early phase of carcinogenesis and cancer progression and is involved in aspects other than simply conferring therapeutic resistance.

Highly proliferative cells, such as cancer cells, possess fundamentally different metabolic activities from quiescent cells [141]. Proliferating cells require abundant nutrients for the synthesis of cellular components including lipids, proteins and nucleic acids. We have recently found that the activity of NRF2 is augmented under sustained activation of the PI3K-AKT pathway, which enables NRF2 to activate metabolic genes involved in the glucose and glutamine metabolism and contribute to metabolic reprogramming for cell proliferation [131]. In particular, NRF2 activates the four key enzyme genes of the pentose phosphate pathway, G6PD, PGD, TKT and TALDO1. Direct regulation of these metabolic genes by NRF2 has been confirmed in an independent ChIP-sequencing analysis with an anti-NRF2 antibody [11]. In addition, two types of microRNAs, miR-1 and miR-206, have been shown to regulate these genes under the control of NRF2 [142].

The activation of NRF2 indeed alters metabolite flux, shunting glycolytic intermediates into the pentose phosphate pathway that enhances the purine nucleotide synthesis [131,142]. NRF2 also promotes glutamine metabolism, thereby enhancing glutathione synthesis and lactate production, which is designated as glutaminolysis. Inhibition of the pentose phosphate pathway in NRF2-active cells reduces their cell growth, and conversely, selective activation of the pentose phosphate pathway in the NRF2-inactive cells accelerates their proliferation. This effectively indicates that 
MURAKAMI et al.

facilitation of the pentose phosphate pathway is one of the major mechanisms the NRF2-mediated acceleration of cell proliferation.

\section{4) Mitochondrial function and lifespan}

A line of evidence has suggested a functional association between the KEAP1-NRF2 system and mitochondrial biogenesis and functions [143,144]. The NRF2-mediated regulation of mitochondrial functions is through the control of its substrate availability [143], and also NRF2 target gene products have been shown to participate in healthy mitochondrial maintenance, removing damaged mitochondria and inducing mitochondrial biogenesis [144]. Conversely, mitochondria themselves have been suggested to regulate NRF2 activity. A pool of SKN-1, which is a C. elegans of NRF2, is localized on the mitochondrial outer membrane, and the SKN-1 liberation from the mitochondria elicits the starvation response [145]. Precise understanding of interrelation between NRF2 and mitochondria may give a new insight of the NRF2-mediated regulation in cell proliferation and differentiation.

It should be noted that SKN-1 mediates diet-restriction-induced longevity [146-148], which has recently been shown accomplished by upregulation of collagens [149]. In analogy to the SKN-1 functions, NRF2 activity is positively correlated with longevity in rodents [150]. Therefore, NRF2 appears to govern lifespan extension, and an adequate level of NRF2 activation would contribute to the achievement of healthy aging.

\section{Conclusions}

As accumulating evidence has clarified the novel actions of NRF2 beyond the 
cytoprotection against oxidative stress and xenobiotics, increasing attention has been paid to the impact of the KEAP1-NRF2 system on basic physiological processes, such as cell cycle regulation and energy metabolism. Though the transcriptome analysis has been a powerful approach to clarify the canonical function of NRF2 in cytoprotection, comprehensive information on the transcripts may not be sufficient to decipher the mechanistic aspects of the non-canonical NRF2 functions related to cell proliferation and differentiation. This is because the integrated outputs from the "NRF2 cistrome" are translated into protein modifications or alterations of metabolic flux distribution. Therefore, in addition to the transcriptome analysis, proteomic and metabolomic analyses are promising strategies for deciphering unsolved mechanisms of the non-canonical NRF2 functions.

One of the interesting questions is how NRF2 contributes to stem cell activity, particularly to the maintenance of stem cells. The excessive proliferation of stem cells for self-renewal often leads to aging and exhaustion of stem cells [56,57], while a substantial body of evidence describes that NRF2 activates the proliferation of tissue stem cells $[70,71,74,75]$. Whether the sustained activation of Nrf2 results in the depletion of stem cells or enhances self-renewal without exhaustion needs to be investigated to evaluate the clinical benefits of NRF2 activators in regenerative medicine. Furthermore, the elucidation of the molecular mechanisms underlying the differential roles of NRF2 in the pluripotent stem cells and tissue stem cells represents an interesting challenge.

Another remaining question in the NRF2 functions that have been reviewed in this article is what physiological stimuli employ NRF2 to regulate cell fate determination. A fascinating example is commensal bacteria residing in the alimentary 
MURAKAMI et al.

and respiratory tracts. Recently, bacterial flora has been shown to have a great impact on host health by modulating the activity of the immune system and systemic metabolism [151,152]. Miscellaneous signals or stresses generated from the microbiota may activate NRF2 for modulating proliferation and differentiation processes. How NRF2 activity is influenced by the microbiota and how NRF2 modulates the host cell behavior in response to the microbiota are attractive issues. Answering these questions would add a new layer of complexity to the NRF2-mediated control of cell proliferation and differentiation. 


\section{Acknowledgements}

This work was supported by JSPS KAKENHI Grant Numbers 24390075 (HM), 15K18999 (SM), MEXT KAKENHI Grant Number 23116002 (HM), the Takeda Scientific Foundation (HM), the Core Research for Evolutional Science and Technology from the JST (HM) and the GSK Japan Research Grant (SM). 


\section{References}

[1] Motohashi, H.; Yamamoto M. Nrf2-Keap1 defines a physiologically important stress response mechanism. Trends Mol. Med. 10:549-557; 2004.

[2] Moi, P.; Chan, K.; Asunis, I.; et al. Isolation of NF-E2-related factor 2 (Nrf2), a NF-E2-like basic leucine zipper transcriptional activator that binds to the tandem NF-E2/AP1 repeat of the beta-globin locus control region. Proc. Natl. Acad. Sci. USA 91:9926-9930; 1994.

[3] Itoh, K.; Igarashi, K.; Hayashi, N; et al. Cloning and characterization of a novel erythroid cell-derived $\mathrm{CNC}$ family transcription factor heterodimerizing with the small Maf family proteins. Mol. Cell. Biol. 15:4184-4193; 1995.

[4] Igarashi, K.; Kataoka, K.; Itoh, K.; et al. Regulation of transcription by dimerization of erythroid factor NF-E2 p45 with small Maf proteins. Nature 367:568-572; 1994.

[5] Kataoka, K.; Igarashi, K.; Itoh, K.; et al. Small Maf proteins heterodimerize with Fos and may act as competitive repressors of the NF-E2 transcription factor. Mol. Cell. Biol. 15:2180-2190; 1995.

[6] Motohashi, H.; Katsuoka, F.; Shavit, J. A.; et al. Positive or negative MARE-dependent transcriptional regulation is determined by the abundance of small Maf proteins. Cell 103:865-875; 2000.

[7] Motohashi, H.; Katsuoka, F.; Miyoshi, C.; et al. MafG sumoylation is required for active transcriptional repression. Mol. Cell. Biol. 26:4652-4663; 2006.

[8] Motohashi, H.; Fujita, R.; Takayama, M.; et al. Molecular determinants for small Maf protein control of platelet production. Mol. Cell. Biol. 31:151-162; 2011.

[9] Yamamoto, T,; Kyo, M.; Kamiya, T.; et al. Predictive base substitution rules that 
determine the binding and transcriptional specificity of Maf recognition elements. Genes Cells 11:575-591; 2006.

[10] Kimura, M.; Yamamoto, T.; Zhang, J.; et al. Molecular basis distinguishing the DNA binding profile of Nrf2-Maf heterodimer from that of Maf homodimer. J. Biol. Chem. 282:33681-33690; 2007.

[11]Hirotsu, Y.; Katsuoka, F.; Funayama, R.; et al. Nrf2-MafG heterodimers contribute globally to antioxidant and metabolic networks. Nucleic Acids Res. 40:10228-10239; 2012.

[12]Fujita, R.; Takayama-Tsujimoto, M.; Satoh, H.; et al. NF-E2 p45 is important for establishing normal function of platelets. Mol. Cell. Biol. 33:2659-2670; 2013.

[13]Frilling, R. S.; Bensimon, A.; Tichauer, Y.; et al. Xenobiotic-inducible expression of murine glutathione S-transferase Ya subunit gene is controlled by an electrophile-responsive element. Proc. Natl. Acad. Sci. USA 87:6258-6262; 1990.

[14]Rushmore, T. H.; Pickett, C. B. Transcriptional regulation of the rat glutathione S-transferase Ya subunit gene. Characterization of a xenobiotic-responsive element controlling inducible expression by phenolic antioxidants. J. Biol. Chem. $265: 14648-14653 ; 1990$.

[15]Rushmore, T.H.; Morton, M.R.; Pickett, C. B. The antioxidant responsive element. Activation by oxidative stress and identification of the DNA consensus sequence required for functional activity. J. Biol. Chem. 266:11632-11639; 1991.

[16]D'Autréaux, B.; Toledano, M. B. ROS as signaling molecules: mechanisms that generate specificity in ROS homeostasis. Nat. Rev. Mol. Cell Biol. 8:813-824; 2007.

[17] Ye, Z. W.; Zhang, J.; Townsend, D. W.; et al. Oxidative stress, redox regulation and diseases of cellular differentiation. Biochim. Biophys. Acta. 1850:1607-1621; 
MURAKAMI et al.

2015.

[18]Ray, P. D.; Huang, B. W.; Tsuji, Y. Reactive oxygen species (ROS) homeostasis and redox regulation in cellular signaling. Cell. Signal. 24:981-990; 2012.

[19]Forman, H. J.; Maiorino, M.; Ursini, F. Signaling functions of reactive oxygen species. Biochemistry 49:835-842; 2010.

[20] Brigelius-Flohé, R; Flohé, L. Basic principles and emerging concepts in the redox control of transcription factors. Antioxid. Redox Signal. 15:2335-2381; 2011.

[21]Hayes, J. D.; Dinkova-Kostova, A. T. The Nrf2 regulatory network provides an interface between redox and intermediary metabolism. Trends Biochem. Sci. 39:199-218; 2014.

[22]Zaidi, M. Skeletal remodeling in health and disease. Nat. Med. 13:791-801; 2007.

[23] Garrett, I. R.; Boyce, B. F.; Oreffo, R. O.; et al. Oxygen-derived free radicals stimulate osteoclastic bone resorption in rodent bone in vitro and in vivo. J. Clin. Invest. 85:632-639; 1990.

[24]Lean, J. M.; Davies, J. T.; Fuller, K.; et al. A crucial role for thiol antioxidants in estrogen-deficiency bone loss. J. Clin. Invest. 112:915-923; 2003.

[25]Hyeon, S.; Lee, H.; Yang, Y.; et al. Nrf2 deficiency induces oxidative stress and promotes RANKL-induced osteoclast differentiation. Free Radic. Biol. Med. 65:789-799; 2013.

[26]Kanzaki, H.; Shinohara, F.; Kajiya, M.; et al. The Keap1/Nrf2 protein axis plays a role in osteoclast differentiation by regulating intracellular reactive oxygen species signaling. J. Biol. Chem. 288:23009-23020; 2013.

[27]Ducy, P.; Zhang, R.; Geoffroy, V.; et al. Osf2/Cbfa1: a transcriptional activator of osteoblast differentiation. Cell 89:747-754; 1997. 
[28] Komori, T.; Yagi, H.; Nomura, S.; et al. Targeted disruption of Cbfal results in a complete lack of bone formation owing to maturational arrest of osteoblasts. Cell 89:755-764; 1997.

[29]Hinoi, E.; Fujimori, S.; Wang, L.; et al. Nrf2 negatively regulates osteoblast differentiation via interfering with Runx2-dependent transcriptional activation. $J$. Biol. Chem. 281:18015-18024; 2006.

[30] Inada, M.; Yasui, T.; Nomura, S.; et al. Maturational disturbance of chondrocytes in Cbfal-deficient mice. Dev. Dyn. 214:279-290; 1999.

[31]Hinoi, E.; Takarada, T.; Fujimori, S.; et al. Nuclear factor p45-related factor 2 negatively regulates chondrogenesis. Bone 40:337-344; 2007.

[32]Park, C. K.; Lee, Y.; Kim, K. H.; et al. Nrf2 is a novel regulator of bone acquisition. Bone 63:36-46; 2014.

[33]Lacey, D. L.; Timms, E.; Tan, H. L.; et al. Osteoprotegerin ligand is a cytokine that regulates osteoclast differentiation and activation. Cell 93:165-176; 1998.

[34]Emery, J. G.; McDonnell, P.; Burke, M. B.; et al. Osteoprotegerin is a receptor for the cytotoxic ligand TRAIL. J. Biol. Chem. 273:14363-14367; 1998.

[35] Jia, D.; Gaddy, D.; Suva, L. J.; et al. Rapid loss of bone mass and strength in mice after abdominal irradiation. Radiat. Res. 176:624-635; 2011.

[36]Kondo, H.; Yumoto, K.; Alwood, J. S.; et al. Oxidative stress and gamma radiation-induced cancellous bone loss with musculoskeletal disuse. J. Appl. Physiol. 108:152-161; 2010.

[37]Rana, T.; Schultz, M. A.; Freeman, M. L.; et al. Loss of Nrf2 accelerates ionizing radiation-induced bone loss by upregulating RANKL. Free Radic. Biol. Med. $53: 2298-2307 ; 2012$. 
MURAKAMI et al.

[38]Pi, J.; Leung, L.; Xue, P.; et al. Deficiency in the nuclear factor E2-related factor-2 transcription factor results in impaired adipogenesis and protects against diet-induced obesity. J. Biol. Chem. 285:9292-9300; 2010.

[39]Hou, Y.; Xue, P.; Bai, Y.; et al. Nuclear factor erythroid-derived factor2-related factor 2 regulates transcription of CCAAT/enhancer-binding protein $\beta$ during adipogenesis. Free Radic. Biol. Med. 52:462-472; 2012.

[40] Cristancho, A. G.; Lazar, M. A. Forming functional fat: a growing understanding of adipocyte differentiation. Nat. Rev. Mol. Cell Biol. 12:722-734; 2011.

[41] Xue, P.; Hou, Y.; Chen, Y.; et al. Adipose deficiency of Nrf2 in $o b / o b$ mice results in severe metabolic syndrome. Diabetes 62:845-854; 2013.

[42]Xu, J.; Kulkarni, S. R.; Donepudi, A. C.; et al. Enhanced Nrf2 activity worsens insulin resistance, impairs lipid accumulation in adipose tissue, and increases hepatic steatosis in leptin-deficient mice. Diabetes 61:3208-3218; 2012.

[43] Shin, S.; Wakabayashi, N.; Misra, V.; et al. NRF2 modulates aryl hydrocarbon receptor signaling: influence on adipogenesis. Mol. Cell. Biol. 27:7188-7197; 2007.

[44] Chorley, B. N.; Campbell, M. R.; Wang, X.; et al. Identification of novel NRF2-regulated genes by ChIP-Seq: influence on retinoid $\mathrm{X}$ receptor alpha. Nucleic Acids Res. 40:7416-7429; 2012.

[45]Lefebvre, B.; Benomar, Y.; Guédin A.; et al. Proteasomal degradation of retinoid X receptor alpha reprograms transcriptional activity of $\operatorname{PPAR} \gamma$ in obese mice and humans. J. Clin. Invest. 120:1454-1468; 2010.

[46]Uruno, A.; Furusawa, Y.; Yagishita, Y.; et al. The Keap1-Nrf2 system prevents onset of diabetes mellitus. Mol. Cell. Biol. 33:2996-3010; 2013.

[47] Yagishita, Y.; Fukutomi, T.; Sugawara, A.; et al. Nrf2 protects pancreatic $\beta$-cells 
from oxidative and nitrosative stress in diabetic model mice. Diabetes 63:605-618; 2014.

[48] Drevet J. R. The antioxidant glutathione peroxidase family and spermatozoa: a complex story. Mol. Cell. Endocrinol. 250:70-79; 2006.

[49] Agarwal, A.; Virk, G.; Ong, C.; et al. Effect of oxidative stress on male reproduction. World J. Mens Health 32:1-17; 2014.

[50]Nakamura, B. N.; Lawson, G.; Chan, J. Y.; et al. Knockout of the transcription factor NRF2 disrupts spermatogenesis in an age-dependent manner. Free Radic. Biol. Med. 49:1368-1379; 2010.

[51] Yu, B.; Lin, H.; Yang, L.; et al. Genetic variation in the Nrf2 promoter associates with defective spermatogenesis in humans. J. Mol. Med. 90:1333-1342; 2012.

[52] Suzuki, T.; Shibata, T.; Takaya, K.; et al. Regulatory nexus of synthesis and degradation deciphers cellular Nrf2 expression levels. Mol. Cell. Biol. $33: 2402-2412 ; 2013$.

[53]Cho, H. Y.; van Houten, B.; Wang, X.; et al. Targeted deletion of nrf2 impairs lung development and oxidant injury in neonatal mice. Antioxid. Redox Signal. 17:1066-1082; 2012.

[54]Huebner, A. J.; Dai, D.; Morasso, M.; et al. Amniotic fluid activates the nrf2/keap1 pathway to repair an epidermal barrier defect in utero. Dev. Cell 23:1238-1246; 2012.

[55]Rossi, D. J.; Bryder, D.; Seita, J.; et al. Deficiencies in DNA damage repair limit the function of haematopoietic stem cells with age. Nature 447:725-730; 2007.

[56] Orford, K. W.; Scadden, D. T. Deconstructing stem cell self-renewal: genetic insights into cell cycle regulation. Nat. Rev. Genet. 9:115-128; 2008. 
MURAKAMI et al.

[57]Beerman, I.; Bock, C.; Garrison, B. S.; et al. Proliferation-dependent alterations of the DNA methylation landscape underlie hematopoietic stem cell aging. Cell Stem Cell 12:413-425; 2013.

[58]Cheung, T. H.; Rando, T. A. Molecular regulation of stem cell quiescence. Nat. Rev. Mol. Cell Biol. 14:329-340; 2013.

[59] Wang, K.; Zhang, T.; Dong, Q.; et al. Redox homeostasis: the linchpin in stem cell self-renewal and differentiation. Cell Death Dis. 4:e537; 2013.

[60]Le Belle, J. E.; Orozco, N. M.; Paucar, A. A.; et al. Proliferative neural stem cells have high endogenous ROS levels that regulate self-renewal and neurogenesis in a PI3K/Akt-dependant manner. Cell Stem Cell 8:59-71; 2011.

[61] Myant, K. B.; Cammareri, P.; McGhee, E. J.; et al. ROS production and NF-кB activation triggered by RAC1 facilitate WNT-driven intestinal stem cell proliferation and colorectal cancer initiation. Cell Stem Cell 12:761-773; 2013.

[62]Jang, Y. Y.; Sharkis, S. J. A low level of reactive oxygen species selects for primitive hematopoietic stem cells that may reside in the low-oxygenic niche. Blood 110:3056-3063; 2007.

[63] Miyamoto, K.; Araki, K. Y.; Naka, K; et al. Foxo3a is essential for maintenance of the hematopoietic stem cell pool. Cell Stem Cell 1:101-112; 2007.

[64]Ito, K.; Hirao, A.; Arai, F.; et al. Reactive oxygen species act through p38 MAPK to limit the lifespan of hematopoietic stem cells. Nat. Med. 12:446-451; 2006.

[65]Hochmuth, C. E.; Biteau, B.; Bohmann, D.; et al. Redox regulation by Keap1 and Nrf2 controls intestinal stem cell proliferation in Drosophila. Cell Stem Cell 8:188-199; 2011.

[66]Forman, H. J.; Augusto, O.; Brigelius-Flohe, R.; et al. Even free radicals follow 
some rules: a guide to free radical research terminology and methodology. Free Radic. Biol. Med. 78:233-235; 2015.

[67] Kalyanaraman, B.; Darley-Usmar, V.; Davies, K. J.; et al. Measuring reactive oxygen and nitrogen species with fluorescent probes: challenges and limitations. Free Radic. Biol. Med. 52:1-6; 2012.

[68]Rock, J. R.; Onaitis, M. W.; Rawlins, E. L.; et al. Basal cells as stem cells of the mouse trachea and human airway epithelium. Proc. Natl. Acad. Sci. USA $106: 12771-12775 ; 2009$.

[69]Hegab, A. E.; Ha, V. L.; Gilbert, J. L.; et al. Novel stem/progenitor cell population from murine tracheal submucosal gland ducts with multipotent regenerative potential. Stem Cells 29:1283-1293; 2011.

[70]Paul, M. K.; Bisht, B.; Darmawan, D. O.; et al. Dynamic changes in intracellular ROS levels regulate airway basal stem cell homeostasis through Nrf2-dependent Notch signaling. Cell Stem Cell 15:199-214; 2014.

[71]Jang, J.; Wang, Y.; Kim, H.S.; et al. Nrf2, a regulator of the proteasome, controls self-renewal and pluripotency in human embryonic stem cells. Stem Cells $32: 2616-2625 ; 2014$.

[72]Chargé, S. B.; Rudnicki, M. A. Cellular and molecular regulation of muscle regeneration. Physiol. Rev. 84:209-238; 2004.

[73] Kuang, S.; Rudnicki, M. A. The emerging biology of satellite cells and their therapeutic potential. Trends Mol. Med. 14:82-91; 2008.

[74]Al-Sawaf, O.; Fragoulis, A.; Rosen, C.; et al. Nrf2 augments skeletal muscle regeneration after ischaemia-reperfusion injury. J. Pathol. 234:538-547; 2014.

[75]Kärkkäinen, V.; Pomeshchik, Y.; Savchenko, E.; et al. Nrf2 regulates neurogenesis 
MURAKAMI et al.

and protects neural progenitor cells against A $\beta$ toxicity. Stem Cells 32:1904-1916; 2014.

[76]Zhao, F.; Wu, T.; Lau, A.; et al. Nrf2 promotes neuronal cell differentiation. Free Radic. Biol. Med. 47:867-879; 2009.

[77] Wilson, A.; Laurenti, E.; Oser, G.; et al. Hematopoietic stem cells reversibly switch from dormancy to self-renewal during homeostasis and repair. Cell 135:1118-1129; 2008.

[78]Sun, J.; Ramos, A.; Chapman, B.; et al. Clonal dynamics of native haematopoiesis. Nature 514:322-327; 2014.

[79]Essers, M. A.; Offner, S.; Blanco-Bose, W. E.; et al. IFNa activates dormant haematopoietic stem cells in vivo. Nature 458:904-908; 2009.

[80]Baldridge, M. T.; King, K. Y.; Boles, N. C.; et al. Quiescent haematopoietic stem cells are activated by IFN- $\gamma$ in response to chronic infection. Nature 465:793-797; 2010.

[81] Tothova, Z.; Kollipara, R.; Huntly, B. J.; et al. FoxOs are critical mediators of hematopoietic stem cell resistance to physiologic oxidative stress. Cell 128:325-339; 2007.

[82]Parmar, K.; Mauch, P.; Vergilio, J. A.; et al. Distribution of hematopoietic stem cells in the bone marrow according to regional hypoxia. Proc. Natl. Acad. Sci. USA 104:5431-5436; 2007.

[83]Chen, C.; Liu, Y.; Liu, R.; et al. TSC-mTOR maintains quiescence and function of hematopoietic stem cells by repressing mitochondrial biogenesis and reactive oxygen species. J. Exp. Med. 205:2397-2408; 2008.

[84] Merchant, A. A.; Singh, A.; Matsui, W.; et al. The redox-sensitive transcription 
factor Nrf2 regulates murine hematopoietic stem cell survival independently of ROS levels. Blood 118:6572-6579; 2011.

[85] Tsai, J. J.; Dudakov, J. A.; Takahashi, K.; et al. Nrf2 regulates haematopoietic stem cell function. Nat. Cell Biol. 15:309-316; 2013.

[86] Sugiyama, T.; Kohara, H.; Noda, M.; et al. Maintenance of the hematopoietic stem cell pool by CXCL12-CXCR4 chemokine signaling in bone marrow stromal cell niches. Immunity 25:977-988; 2006.

[87] Kim, J. H.; Thimmulappa, R. K.; Kumar, V.; et al. NRF2-mediated Notch pathway activation enhances hematopoietic reconstitution following myelosuppressive radiation. J. Clin. Invest. 124:730-741; 2014.

[88] Murakami, S.; Shimizu, R.; Romeo, P. H.; et al. Keap1-Nrf2 system regulates cell fate determination of hematopoietic stem cells. Genes Cells 19:239-253; 2014.

[89]Bobilev, I.; Novik, V.; Levi, I.; et al. The Nrf2 transcription factor is a positive regulator of myeloid differentiation of acute myeloid leukemia cells. Cancer Biol. Ther. 11:317-329; 2011.

[90]Lee, J. M.; Chan, K.; Kan, Y. W.; et al. Targeted disruption of Nrf2 causes regenerative immune-mediated hemolytic anemia. Proc. Nat. Acad. Sci. USA 101:9751-9756; 2004.

[91] Kawatani, Y.; Suzuki, T.; Shimizu, R.; et al. Nrf2 and selenoproteins are essential for maintaining oxidative homeostasis in erythrocytes and protecting against hemolytic anemia. Blood 117:986-996; 2011.

[92] Motohashi, H.; Kimura, M.; Fujita, R.; et al. NF-E2 domination over Nrf2 promotes ROS accumulation and megakaryocytic maturation. Blood 115:677-686; 2010. 
MURAKAMI et al.

[93] Moi. P.; Chan, K.; Asunis, I.; et al. Isolation of NF-E2-related factor 2 (Nrf2), a NF-E2-like basic leucine zipper transcriptional activator that binds to the tandem NF-E2/AP-1 repeat of the beta-globin locus control region. Proc. Natl. Acad. Sci. USA 91:9926-9930; 1994.

[94]Chan, K.; Lu, R.; Chang, J. C.; et al. NRF2, a member of the NFE2 family of transcription factors, is not essential for murine erythropoiesis, growth, and development. Proc. Natl. Acad. Sci. USA 93:13943-13948; 1996.

[95]Itoh, K.; Chiba, T.; Takahashi, S.; et al. An Nrf2/small Maf heterodimer mediates the induction of phase II detoxifying enzyme genes through antioxidant response elements. Biochem. Biophys. Res. Commun. 236:313-322; 1997.

[96] Kuroha, T.; Takahashi, S.; Komeno, T.; et al. Ablation of Nrf2 function does not increase the erythroid or megakaryocytic cell lineage dysfunction caused by p45 NF-E2 gene disruption. J. Biochem. 123:376-379; 1998.

[97] Shivdasani, R. A.; Rosenblatt, M. F.; Zucker-Fanklin, D.; et al. Transcription factor NF-E2 is required for platelet formation independent of the actions of thrombopoietin/MGDF in megakaryocyte development. Cell 81:695-704; 1995.

[98]Takayama, M.; Fujita, R.; Suzuki, M.; et al. Genetic analysis of hierarchical regulation for Gata1 and NF-E2 p45 gene expression in megakaryopoiesis. Mol. Cell. Biol. 30:2668-2680; 2010.

[99]Andrews, N. C.; Erdjument-Bromage, H.; Davidson, M. B.; et al. Erythroid transcription factor NF-E2 is a haematopoietic-specific basic-leucine zipper protein. Nature 362:722-728; 1993.

[100] McCrann, D. J.; Eliades, A.; Makitalo, M.; et al. Differential expression of NADPH oxidases in megakaryocytes and their role in polyploidy. Blood 
114:1243-1249; 2009.

[101] Caterina, J. J.; Donze, D.; Sun, C. W.; et al. Cloning and functional characterization of LCR-F1: a bZIP transcription factor that activates erythroid-specific, human globin gene expression. Nucleic Acids Res. 22:2383-2391; 1994.

[102] Kobayashi, A.; Ito, E.; Toki, T.; et al. Molecular cloning and functional characterization of a new Cap'n' collar family transcription factor Nrf3. J. Biol. Chem. 274:6443-6452; 1999.

[103] Oyake, T.; Itoh, K.; Motohashi, H.; et al. Bach proteins belong to a novel family of BTB-basic leucine zipper transcription factors that interact with MafK and regulate transcription through the NF-E2 site. Mol. Cell. Biol. 16:6083-6095, 1996.

[104] Sun, J.; Hoshino, H.; Takaku, K.; et al. Hemoprotein Bach1 regulates enhancer availability of heme oxygenase-1 gene. EMBO J. 21:5216-5224; 2002.

[105] Katsuoka, F.; Motohashi, H.; Tamagawa, Y.; et al. Small Maf compound mutants display central nervous system neuronal degeneration, aberrant transcription, and Bach protein mislocalization coincident with myoclonus and abnormal startle response. Mol. Cell. Biol. 23:1163-1174; 2003.

[106] Tsujita, T.; Peirce, V.; Baird, L.; et al. Transcription factor Nrf1 negatively regulates the cystine/glutamate transporter and lipid-metabolizing enzymes. Mol. Cell. Biol. 34:3800-3816; 2014.

[107] Itoh-Nakadai, A.; Hikota, R.; Muto, A.; et al. The transcription repressors Bach2 and Bach1 promote B cell development by repressing the myeloid program. Nat. Immunol. 15:1171-1180; 2014. 
MURAKAMI et al.

[108] Muto, A.; Hoshino, H.; Madisen, L.; et al. Identification of Bach2 as a B-cell-specific partner for small maf proteins that negatively regulate the immunoglobulin heavy chain gene 3' enhancer. EMBO J. 17:5734-5743; 1998.

[109] Singh, A.; Settleman, J. EMT, cancer stem cells and drug resistance: an emerging axis of evil in the war on cancer. Oncogene 29:4741-4751; 2010.

[110] Beck, B.; Blanpain, C. Unravelling cancer stem potential. Nat. Rev. Cancer $13: 727-738 ; 2013$.

[111] Cancer Genome Atlas Research Network. Comprehensive genomic characterization of squamous cell lung cancers. Nature 489:519-525; 2012.

[112] Kandoth, C.; McLellan, M. D.; Vandin, F.; et al. Mutational landscape and significance across 12 major cancer types. Nature 502:333-339; 2013.

[113] Cancer Genome Atlas Research Network. Comprehensive molecular profiling of lung adenocarcinoma. Nature 511:543-550; 2014.

[114] Padmanabhan, B.; Tong, K. I.; Ohta, T.; et al. Structural basis for defects of Keap1 activity provoked by its point mutations in lung cancer. Mol. Cell 21:689-700; 2006.

[115] Ohta, T.; Iijima, K.; Miyamoto, M.; et al. Loos of Keap1 function activates Nrf2 and provides advantages for lung cancer cell growth. Cancer Res. 68:1303-1309; 2008.

[116] Shibata, T.; Ohta, T.; Tong, T. I.; et al. Cancer related mutations in NRF2 impair its recognition by Keap1-Cul3 E3 ligase and promote malignancy. Proc. Natl. Acad. Sci. USA 105:13568-13573; 2008.

[117] Solis, L. M.; Behrens, C.; Suraokar, M.; et al. Nrf2 and Keap1 abnormalities in non-small cell lung carcinoma and association with clinicopathologic features. Clin. 
Cancer Res. 16:3743-3753; 2010.

[118] Inoue, D.; Suzuki, T.; Mitsuishi, Y.; et al. Accumulation of p62/SQSTM1 is associated with poor prognosis in patients with lung adenocarcinoma. Cancer Sci. 103:760-766; 2012.

[119] Onodera, Y.; Motohashi, H.; Takagi, K.; et al. NRF2 immunolocalization in human breast cancer patients as a prognostic factor. Endocr. Relat. Cancer $21: 241-252 ; 2014$.

[120] Kanamori, M.; Higa, T.; Sonoda, Y.; et al. Activation of the NRF2 pathway and its impact on the prognosis of anaplastic glioma patients. Neuro. Oncol. $17: 555-565 ; 2015$.

[121] Vescovi, A. L.; Galli, R.; Reynolds, B. A. Brain tumour stem cells. Nat. Rev. Cancer 6:425-436; 2006.

[122] Persano, L.; Rampazzo, E.; Basso, G.; et al. Glioblastoma cancer stem cells: role of the microenvironment and therapeutic targeting. Biochem. Pharmacol. 85:612-622; 2013.

[123] Zhu, J.; Wang, H.; Sun, Q.; et al. Nrf2 is required to maintain the self-renewal of glioma stem cells. BMC Cancer 13:380; 2013.

[124] Zhu, J.; Wang, H.; Ji, X.; et al. Differential Nrf2 expression between glioma stem cells and non-stem-like cells in glioblastoma. Oncol. Lett. 7:693-698; 2014.

[125] Singer, E.; Judkins, J.; Salomonis, N.; et al. Reactive oxygen species-mediated therapeutic response and resistance in glioblastoma. Cell Death Dis. 6:e1601; 2015.

[126] Del Vecchio, C. A.; Feng, Y.; Sokol, E. S.; et al. De-differentiation confers multidrug resistance via noncanonical PERK-Nrf2 signaling. PLoS Biol. 12:e1001945; 2014. 
MURAKAMI et al.

[127] Beyer, T. A.; Xu, W.; Teupser, D.; et al. Impaired liver regeneration in Nrf2 knockout mice: role of ROS-mediated insulin/IGF-1 resistance. EMBO J. 27:212-223, 2008.

[128] Schwabe, R. F.; Brenner, D. A. Mechanisms of liver injury. I. TNF- $\alpha$-induced liver injury: role of IKK, JNK, and ROS pathways. Am. J. Physiol. Gastrointest. Liver Physiol. 290:G583-G589; 2006.

[129] Wakabayashi, N.; Shin, S.; Slocum, S. L.; et al. Regulation of notch1 signaling by nrf2: implications for tissue regeneration. Sci. Signal. 3:ra52; 2010.

[130] Shirasaki, K.; Taguchi, K.; Unno, M.; et al. NF-E2-related factor 2 promotes compensatory liver hypertrophy after portal vein branch ligation in mice. Hepatology 59:2371-2382; 2014.

[131] Mitsuishi, Y.; Taguchi, K.; Kawatani, Y.; et al. Nrf2 redirects glucose and glutamine into anabolic pathways in metabolic reprogramming. Cancer Cell 22:66-79; 2012.

[132] Taguchi, K.; Hirano, I.; Itoh, T.; et al. Nrf2 enhances cholangiocyte expansion in Pten-deficient livers. Mol. Cell. Biol. 34:900-913; 2014.

[133] Rada, P.; Rojo, A. I.; Chowdhry, S.; et al. SCF/ $\beta$-TrCP promotes glycogen synthase kinase 3-dependent degradation of the Nrf2 transcription factor in a Keap1-independent manner. Mol. Cell. Biol. 31:1121-1133; 2011.

[134] Rojo, A. I.; Medina-Campos, O. N.; Rada, P.; et al. Signaling pathways activated by the phytochemical nordihydroguaiaretic acid contribute to a Keap1-independent regulation of Nrf2 stability: Role of glycogen synthase kinase-3. Free Radic. Biol. Med. 52:473-487; 2012.

[135] Malhotra, D.; Portales-Casamar, E.; Singh, A.; et al. Global mapping of binding 
sites for Nrf2 identifies novel targets in cell survival response through ChIP-Seq profiling and network analysis. Nucleic Acids Res. 38:5718-5734; 2010.

[136] Zou, Y.; Hu, M.; Lee, J.; et al. Nrf2 is essential for timely M-phase entry of replicating hepatocytes during liver regeneration. Am. J. Physiol. Gastrointest. Liver Physiol. 308:G262-G268; 2015.

[137] Reddy, N. M.; Kleeberger, S. R.; Bream, J. H.; et al. Genetic disruption of the Nrf2 compromises cell-cycle progression by impairing GSH-induced redox signaling. Oncogene 27:5821-5832; 2008.

[138] Kellogg, D. R. Wee1-dependent mechanisms required for coordination of cell growth and cell division. J. Cell Sci. 116:4883-4890; 2003.

[139] Fisher, D.; Krasinska, L.; Coudreuse, D.; et al. Phosphorylation network dynamics in the control of cell cycle transitions. J. Cell Sci. 125:4703-4711; 2012.

[140] DeNicola, G. M.; Karreth, F. A.; Humpton, T. J.; et al. Oncogene-induced Nrf2 transcription promotes ROS detoxification and tumorigenesis. Nature 475:106-109; 2011.

[141] DeBerardinis, R. J.; Lum, J. J.; Hatzivassiliou, G.; et al. The biology of cancer: metabolic reprogramming fuels cell growth and proliferation. Cell Metab. 7:11-20; 2008.

[142] Singh, A.; Happel, C.; Manna, S. K.; et al. Transcription factor NRF2 regulates miR-1 and miR-206 to drive tumorigenesis. J. Clin. Invest. 123:2921-2934; 2013.

[143] Holmström, K. M.; Baird, L.; Zhang, Y; et al. Nrf2 impacts cellular bioenergetics by controlling substrate availability for mitochondrial respiration. Biol. Open 2:761-770; 2013.

[144] Itoh, K.; Ye, P.; Matsumiya, T.; et al. Emerging functional cross-talk between 
MURAKAMI et al.

the Keap1-Nrf2 system and mitochondria. J. Clin. Biochem. Nutr. 56:91-97; 2015.

[145] Paek, J.; Lo, J. Y.; Narasimhan, S. D.; et al. Mitochondrial SKN-1/Nrf mediates a conserved starvation response. Cell Metab. 16:526-537; 2012.

[146] An, J. H.; Blackwell, T. K. SKN-1 links C. elegans mesendodermal specification to a conserved oxidative stress response. Genes Dev. 17:1882-1893; 2003.

[147] Bishop, A. N.; Guarente, L. Two neurons mediate diet-restriction-induced longevity in C. elegans. Nature 447:545-549; 2007.

[148] Tullet, J. M.; Hertweck, M.; An, J. H.; et al. Direct inhibition of the longevity-promoting factor SKN-1 by insulin-like signaling in C. elegans. Cell $132: 1025-1038 ; 2008$.

[149] Ewald, C. Y.; Landis, J. N.; Abate, J. P.; et al. Dauer-independent insulin/IGF-1-signalling implicates collagen remodeling in longevity. Nature 519:97-101; 2015.

[150] Lewis, K. N.; Wason, E.; Edrey, Y. H.; et al. Regulation of Nrf2 signaling and longevity in naturally long-lived rodents. Proc. Natl. Acad. Sci. USA 112:3722-3737; 2015.

[151] Tremaroli, V.; Bäckhed, F. Functional interactions between the gut microbiota and host metabolism. Nature 489:242-249; 2012.

[152] Maynard, C. L.; Elson, C. O.; Hatton, R. D.; et al. Reciprocal interactions of the intestinal microbiota and immune system. Nature 489:231-241; 2012. 


\section{Figure Legends}

Figure 1. Downstream effectors of the NRF2-dependent regulation in cell proliferation and differentiation.

A. Elevation of canonical target genes of NRF2. NRF2 modifies cell activity by controlling intracellular levels of oxidative stress, which comprises an important regulatory axis of osteoclast differentiation (i) and maintenance of Drosophila ISCs (ii). B. Novel NRF2 target genes that regulate cell proliferation and differentiation. NRF2-mediated induction of PPAR $\gamma$ and CEBP $\beta$ promotes adipocyte differentiation (i), whereas NRF2-mediated induction of RXR $\alpha$ inhibits adipocyte differentiation (ii). Enhanced NOTCH signaling due to NRF2 activation impacts on the maintenance and proliferation of stem cells and liver regeneration (iii). CXCR4 is indispensable for the maintenance of HSCs, and MyoD is a major regulator of SCs during skeletal muscle regeneration. In NRF2 deficiency, decreased expression levels of each factor leads to impairment of stem cell activity (iv).

ABSC, airway basal stem cell; HSC, hematopoietic stem cell; ISC, intestinal stem cell; MSC, mesenchymal stem cell; SC, satellite cell. 
Table 1. NRF2 impacts on various cell type

\begin{tabular}{cc}
\hline Physiology & Cell Type \\
\hline Bone Homeostasis & Osteoclast \\
& Osteoblast \\
& Osteoclast/Osteoblast \\
& Osteoclast/Osteoblast \\
\hline Adipogenesis & Adipocyte \\
& White Adipose Tissue (WATs) \\
& White Adipose Tissue (WATs) \\
& Adipocyte \\
\hline Stem Cell Activity & Intestinal Stem Cells (ISCs) \\
& Airway Basal Stem Cells (ABSCs) \\
& Embryonic Stem Cells (ESCs) \\
& Satellite Cells (SCs) \\
& Neuronal Progenitor Cells (NPCs) \\
& Hematopoietic Stem Cells (HSCs) \\
& HSCs \\
& HSCs \\
\hline Liver Regeneration & Glioma Stem Cells (GSCs) \\
\hline & Hepatocytes \\
& Liver \\
\hline Energy Metaolism & Hepatocytes \\
& Liver \\
\hline Lifespan & Cancer Cells and MEFs \\
& Cancer Cells \\
\hline & C. elegans \\
\hline
\end{tabular}


A Regulation of intracellular oxidative stress

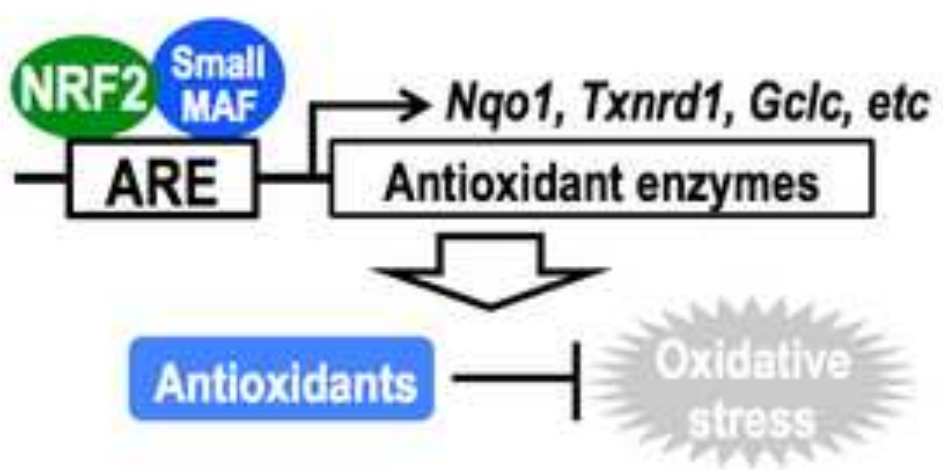

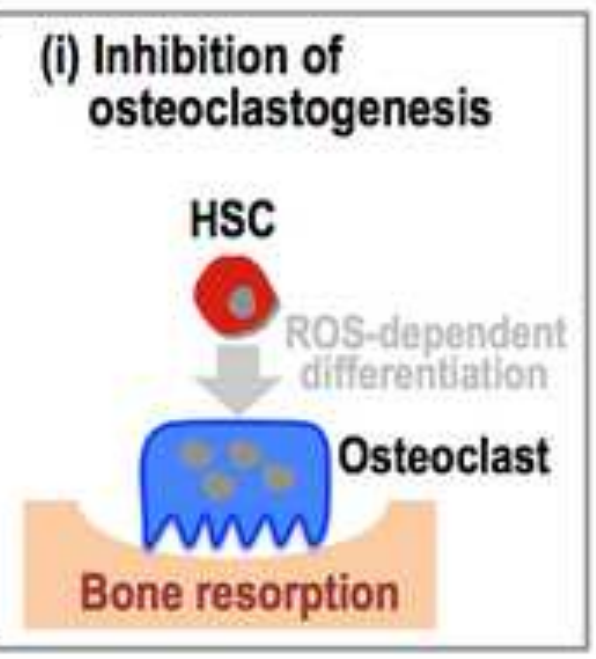

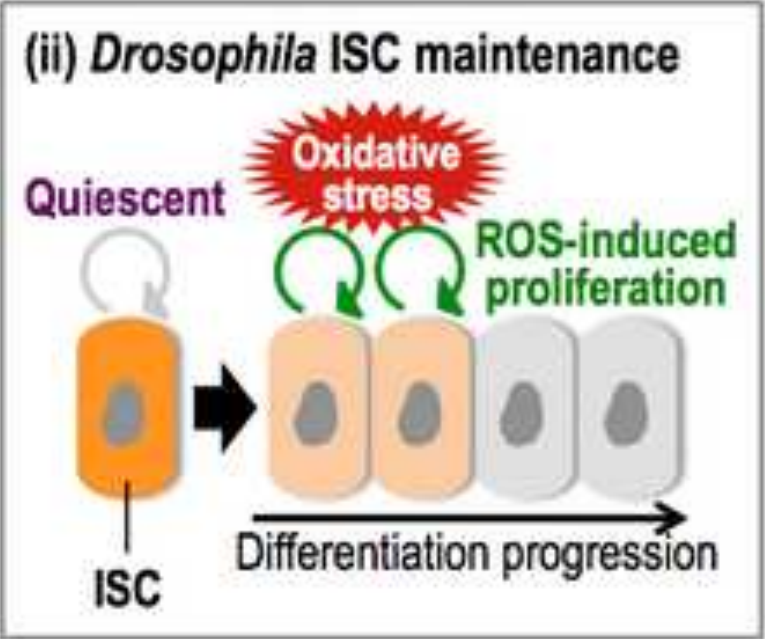

B Induction of cell fate regulators
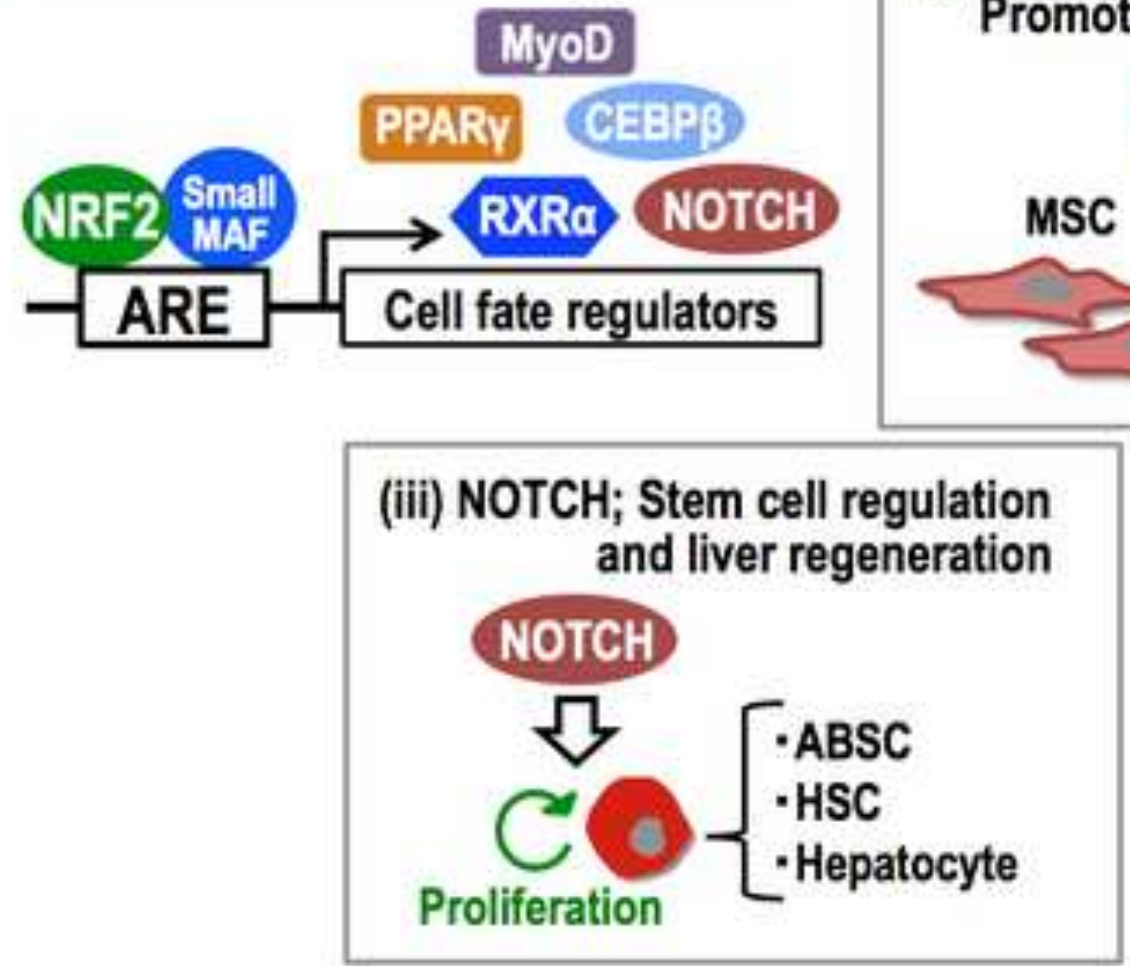

(i) PPARY, CEBP $\beta$;

Promotion of adipogenesis

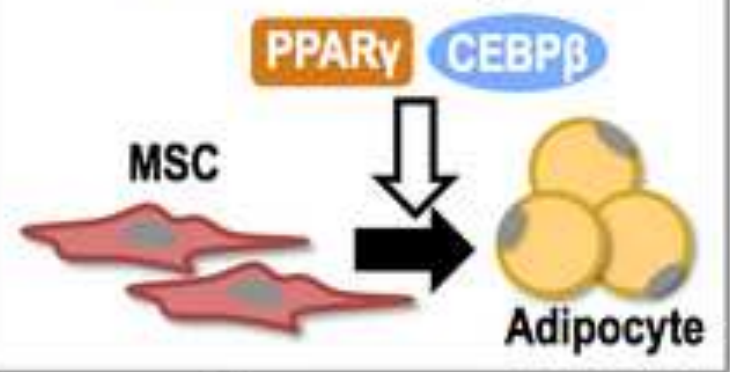

(ii) RXRa; Inhibition of adipogenesis

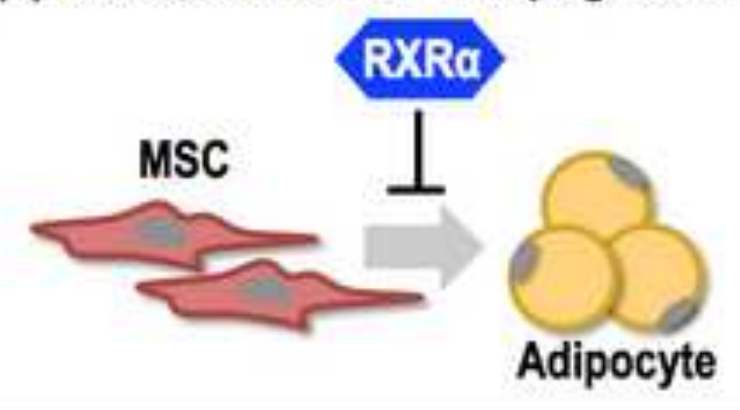

(iv) CXCR4, MyoD; Stem cell regulation
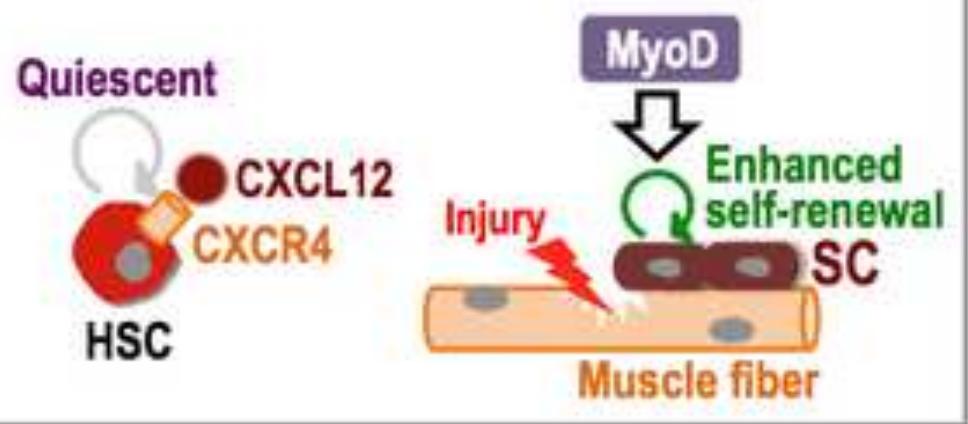